\title{
STATISTICAL REPORT
}

\section{Business and Economic Databases Access in Academic Business Libraries. \\ Donald Schnedeker}

\begin{abstract}
Donald Schnedeker is the Library Director for the Johnson Graduate School of Management Library, Cornell University, Ithaca, NY 14853 (E-mail: dws2@cornell.edu).
\end{abstract}

This column will report on access to business and economic databases and database aggregations ${ }^{\mathrm{i}}$ in academic business libraries. The data comes from surveys of separately housed academic business libraries conducted in the winter 2000 and the fall of 2001. This report covered subscription services. Many libraries now catalog or provide links to free or public Web sites, but this column does not include free or public Web sites that provide business information. The databases accessed in academic business libraries are presented in both alphabetic (Table 1) and ranked (Table 3) lists. Both tables include two years of data for comparison.

Access to electronic business information is changing rapidly. Many articles have been written on the selection and evaluation of electronic resources for libraries. These articles emphasize the collection and evaluation of information on potential database acquisitions and the role of the selector. Davis provides an extensive list of criteria for selecting databases including reputation of authors, publishers and producers, content (scope and breadth), format and special features, technological concerns, access methods, archiving, Licensing limitations, user definition, use capabilities and restrictions, and contractual restrictions. ${ }^{\text {ii }}$ Holleman suggests applying traditional selection criteria, quality, 
relevancy, aesthetic and technical aspects and cost, to electronic resource. ${ }^{\text {iii }}$ Thornton discusses the impact of consortia purchasing on the individual selector. ${ }^{\text {iv }}$ While it is clear that librarians share information across institutions, this sharing is generally considered an informal activity and has not been included in the selection criteria of the evaluation process for electronic resources. As higher education becomes more competitive, schools compete for students and faculty. As librarians it is very useful to know what our colleagues at other schools are doing. This information can enlighten the decision making process as we evaluate new databases. This column provides valuable information on the acceptance of business and economic databases in academic business libraries.

The compiled responses of the libraries participating in this survey provide the librarian an opportunity to benchmark the electronic access to electronic business information offered at their institution. Reviewing the database selections of peer institutions should be one of the tools of the database selector.

\section{The Survey}

In the Fall 2001, thirty-six libraries responded to a survey on library statistics including database access. Thirty-four academic business libraries responded to the 1999/00 survey conducted the previous year. For convenience, the survey combined databases and aggregations into a single list for the respondent. 
In 2000, the respondents were presented with a list of 103 databases and database aggregations. In 2001, this list was updated and expanded to 107 databases and database aggregations. The respondents were asked to answer the following questions:

1. Does your library have access to this database?

2. Do you subscribe to the CD ROM version?

3. Do you access this database through the Web?

4. Do end-users have to pay a fee for access to this database?

5. Do you access this database through a database aggregator, like Dialog, Proquest, and Lexis? If so, which aggregator do you use?

At least one respondent reported accessing all of the 103 electronic information resources listed in the 2000 survey and all but one of the 107 databases listed in the 2001 survey. In 2000 , the survey showed that the thirty-four responding libraries were providing access to an average 37.8 databases. In 2001, the thirty-six responding libraries averaged 40.8 databases from the survey. This demonstrates that academic business libraries are continuing to add database access.

The list of databases and aggregations was based on information reported in the previous survey. Some effort is made to update the names of the databases and aggregations. An alphabetical list of databases and aggregations is included in Table 1. Table 1 also includes the number of responding libraries that reported accessing that resource and comparison data from each database's listing in the 1999/2000 survey. 
Generally, we observe very modest changes in database access from 2000 to 2001. This probably reflects the somewhat conservative nature of academic libraries and the programs that they serve. A few databases made significant gains among the respondents. These included EIU Country Reports growing from 13 subscribers to 23; Netlibrary, from 13 to 23; Valueline from 12 to 21; MCB's Emerald Library from 10 to 19 and finally, the EBSCO Business Source products from 10 to 18 (including Business Source premier and Business Source Elite.) These changes probably reflect the respondents' reactions to forces in the business information marketplace, including price, content and service.

Several electronic resources made their first appearance in the 2000/01 survey. These new additions included Source OECD with 21 subscribers, Sports Business Research Network (19), Gale's Business \& Company Resource Center (16), Forrester (11), Jupiter (9), PsycArticles (8), Multex (7), S\&P's Market Insight (7), Comperio (6). These new additions reflect new products and vendors' efforts to penetrate the academic market.

In addition to adding databases to the survey, an effort was made to include different versions of product offerings. For instance, $\mathrm{ABI} / \mathrm{Inform}$ became $\mathrm{ABI} / \mathrm{Inform}$ Global and ABI/Inform Research and EBSCO Business Source became EBSCO Business Source Premier and EBSCO Business Source Elite. This change was implemented at the request of the respondents who wanted to know which versions of these database products their colleagues were purchasing.

\section{Table 1}




\section{Academic Business Library Statistics, 2000/2001}

\begin{tabular}{|c|c|c|c|}
\hline Databases, 2000/2001 & $\begin{array}{l}(36 \\
\text { Libraries } \\
\text { reporting) }\end{array}$ & Databases, 1999/2000 & $\begin{array}{l}\text { (34 Libraries } \\
\text { reporting) }\end{array}$ \\
\hline Databases & \begin{tabular}{|l|} 
Libraries \\
with \\
Access
\end{tabular} & Databases & $\begin{array}{l}\text { Libraries } \\
\text { with } \\
\text { Access } \\
\end{array}$ \\
\hline 1. ABI/Inform Global & 29 & \begin{tabular}{l|l|}
1 & $\mathrm{ABI} /$ Inform \\
\end{tabular} & 33 \\
\hline \begin{tabular}{l|l}
2 & $\mathrm{ABI} /$ Inform Research \\
\end{tabular} & 7 & 2 Academic Index & 12 \\
\hline \begin{tabular}{l|l}
3 & Academic Index \\
\end{tabular} & 10 & 3 Accounting \& Tax Index & 6 \\
\hline \begin{tabular}{l|l} 
Accounting \& Tax Index \\
\end{tabular} & 6 & 4Amer. Manufacturers Directory & 2 \\
\hline \begin{tabular}{l|l}
5 & Amer. Mfgrs. Directory \\
\end{tabular} & 1 & 5 Article First & 18 \\
\hline \begin{tabular}{l|l}
6 & Article First \\
\end{tabular} & 18 & 6 Bloomberg & 27 \\
\hline 7 Bloomberg Financial Markets & 25 & 7 Bridge & 12 \\
\hline 8 Books $24 \times 7$ & 5 & 8 Business \& Industry & 23 \\
\hline 9 Bridge/Telerate & 8 & 9 Business \& Management Practices & 8 \\
\hline 10 Business \& Company Resource Center (Gale) & 16 & \begin{tabular}{l|l|}
10 Business Index \\
\end{tabular} & 2 \\
\hline 11 Business \& Industry (RDS) & 28 & \begin{tabular}{l|l|}
11 & Business InfoSuite (Gale) \\
\end{tabular} & 2 \\
\hline \begin{tabular}{l|l}
12 & Business \& Mgt. Practices \\
\end{tabular} & 8 & \begin{tabular}{l|l|}
12 & Business Periodicals Index \\
\end{tabular} & 7 \\
\hline \begin{tabular}{l|l}
13 & Business Index (Gale) \\
\end{tabular} & 5 & 13 Business Periodicals Ondisc & 6 \\
\hline 14 Business Infosuite (Gale) & 1 & 14 Career Search & 7 \\
\hline 15 Business Periodicals Index & 7 & \begin{tabular}{l|l|}
15 & $\mathrm{CCH}$ Internet Tax Service \\
\end{tabular} & 18 \\
\hline 16 Business Periodicals Ondisc & 4 & \begin{tabular}{l|l|l|}
16 & Choices II \\
\end{tabular} & 13 \\
\hline 17 CareerSearch & 10 & 17 Citibase & 5 \\
\hline \begin{tabular}{l|l}
18 & CCH Internet Tax Service \\
\end{tabular} & 21 & 18 CompactDisclosure (Primark) & 24 \\
\hline \begin{tabular}{l|l|}
19 & Choices II (Simmons) \\
\end{tabular} & 14 & 19 Corporate Affiliations Directory & 13 \\
\hline 20 Citibase & 4 & 20 Corporate Profiles & 3 \\
\hline 21 Compact Disclosure (Primark) & 21 & 21 CorpTech Directory & 12 \\
\hline \begin{tabular}{|l|l|}
22 & Comperio \\
\end{tabular} & 6 & 22 CRSP & 19 \\
\hline 23 Corp. Affiliations Directory & 11 & 23 DataStar & 3 \\
\hline 24 CRSP & 23 & 24 Datastream & 21 \\
\hline 25 Data Star & 2 & \begin{tabular}{l|l}
25 & DataTimes \\
\end{tabular} & 3 \\
\hline \begin{tabular}{l|l|}
26 & Data Times \\
\end{tabular} & 2 & \begin{tabular}{l|l}
26 & Dialog Web
\end{tabular} & 18 \\
\hline \begin{tabular}{l|l|}
27 & Datastream \\
\end{tabular} & 24 & 27 Dow Jones Interactive & 31 \\
\hline \begin{tabular}{l|l}
28 & Dialog Web \\
\end{tabular} & 13 & 28 Dun's Business Locator & 1 \\
\hline 29 Duns Business Locator & 3 & \begin{tabular}{l|l}
29 & Dun's MM Directory \\
\end{tabular} & 12 \\
\hline 30 Duns MM Directory & 16 & \begin{tabular}{l|l|}
30 & EBSCO's Business Source \\
\end{tabular} & 10 \\
\hline \begin{tabular}{|l|l|}
31 & EBSCO Business Source Elite \\
\end{tabular} & 8 & \begin{tabular}{l|l|}
31 & EconLit \\
\end{tabular} & 28 \\
\hline \begin{tabular}{|l|l|}
32 & EBSCO Business Source Premier \\
\end{tabular} & 10 & \begin{tabular}{|l|l|}
32 & Economic Literature DB \\
\end{tabular} & 7 \\
\hline 33 EconLit & 34 & 33 EIU Country Data & 9 \\
\hline \begin{tabular}{l|l}
34 & Economic Literature DataBase
\end{tabular} & 6 & \begin{tabular}{l|l|}
34 & EIU CountryReports \\
\end{tabular} & 13 \\
\hline \begin{tabular}{l|l}
35 & EIU Business Intl newsletters \\
\end{tabular} & 8 & \begin{tabular}{|l|l|}
35 & EIU News \\
\end{tabular} & 8 \\
\hline 36 EIU Country Data & 13 & 36 EIU Views & 10 \\
\hline \begin{tabular}{|l|l|}
37 & EIU Country reports \\
\end{tabular} & 23 & \begin{tabular}{l|l|}
37 & Extel \\
\end{tabular} & 5 \\
\hline \begin{tabular}{|l|l|}
38 & EIU Views Wire \\
\end{tabular} & 7 & \begin{tabular}{l|l|}
38 & F\&S Index \\
\end{tabular} & 4 \\
\hline 39 Extel & 1 & 39 FARS & 7 \\
\hline 40|F\&S Index (Gale) & 2 & \begin{tabular}{l|l|}
40 & First Call \\
\end{tabular} & 11 \\
\hline
\end{tabular}




\begin{tabular}{|c|c|c|c|}
\hline 41|Factiva & 30 & 41|FIS Online & 18 \\
\hline \begin{tabular}{l|l|}
42 & FARS \\
\end{tabular} & 13 & \begin{tabular}{l|l|l|}
42 & Gale \\
\end{tabular} & 7 \\
\hline \begin{tabular}{l|l|}
43 & First Call \\
\end{tabular} & 9 & \begin{tabular}{l|l}
43 & GAPRO (Primark) \\
\end{tabular} & 9 \\
\hline 44 FIS Online (Moody's) & 22 & 44 Gartner & 6 \\
\hline 45 Forrester & 11 & 45 General Business file & 9 \\
\hline 46 GA Pro (LaserD, Primark) & 9 & 46 Global Access (Primark) & 25 \\
\hline 47 Gale Business Resources & 6 & 47 Global Market Information & 5 \\
\hline \begin{tabular}{l|l}
48 & Gartner Intraweb \\
\end{tabular} & 12 & \begin{tabular}{l|l|}
48 & Hoovers \\
\end{tabular} & 23 \\
\hline $\begin{array}{lll}49 & \text { General Business File } \\
\end{array}$ & 11 & \begin{tabular}{l|l|}
49 & IBES \\
\end{tabular} & 13 \\
\hline 50 Global Access (Primark) & 24 & 50 IFS (International Financial Statistics) & 17 \\
\hline 51 Global Market Information Database (Euromonitor) & 13 & 51 Infotrac Online & 9 \\
\hline 52 Hoovers & 25 & 52 Investext & 20 \\
\hline 53 IBES & 13 & \begin{tabular}{l|l|}
53 & ISI Emerging Markets \\
\end{tabular} & 15 \\
\hline 54 Infotrac (IAC) & 10 & \begin{tabular}{l|l|l|l|l|l}
$54 T O R$ \\
\end{tabular} & 29 \\
\hline 55 Intl. Financial Statistics & 22 & 55 Kalorama (MarketResearch.com) & 8 \\
\hline 56 Investext Plus (Research Bank Web) & 25 & 56 Lexis-Nexis Academic Universe & 29 \\
\hline 57 ISI Emerging Markets & 20 & \begin{tabular}{l|l}
57 & Lexis-Nexis Classic \\
\end{tabular} & 17 \\
\hline 58 JSTOR & 33 & $58 \mathrm{MCB}$ (Emerald) & 10 \\
\hline 59 Jupiter & 9 & \begin{tabular}{l|l|}
59 & Morningstar \\
\end{tabular} & 12 \\
\hline 60 Lexis-Nexis Academic Universe & 34 & \begin{tabular}{l|l}
60 & National Newspaper Index \\
\end{tabular} & 6 \\
\hline $\begin{array}{lll}61 & \text { Lexis-Nexis Classic } \\
\end{array}$ & 24 & \begin{tabular}{l|l} 
61 National Trade Data Bank \\
\end{tabular} & 26 \\
\hline \begin{tabular}{l|l}
62 & Market Insight (S\&P)
\end{tabular} & 7 & \begin{tabular}{l|l}
62 & NBER Working Papers
\end{tabular} & 21 \\
\hline 63 MarketResearch.com & 6 & 63 NetLibrary & 13 \\
\hline \begin{tabular}{l|l|}
64 & MCB-Emerald \\
\end{tabular} & 19 & 64 New York Times & 19 \\
\hline \begin{tabular}{l|l}
65 & Morningstar \\
\end{tabular} & 15 & \begin{tabular}{l|l}
65 & Newspaper Abstracts \\
\end{tabular} & 15 \\
\hline \begin{tabular}{l|l}
66 & Multex \\
\end{tabular} & 7 & $66 \mid$ Nikkei & 4 \\
\hline 67 National Newspaper Index (IAC) & 3 & 67 OECD Index & 5 \\
\hline 68 NBER Working Papers & 27 & \begin{tabular}{l|l|}
68 & OneSource \\
\end{tabular} & 7 \\
\hline 69 NetLibrary & 23 & \begin{tabular}{l|l}
69 & PAIS International
\end{tabular} & 25 \\
\hline 70 Newspaper Abstracts (Proquest) & 16 & 70 Phonedisc & 1 \\
\hline 71 Nikkei & 2 & \begin{tabular}{l|l}
71 & Political Risk \\
\end{tabular} & 10 \\
\hline \begin{tabular}{l|l|}
72 & NY Times \\
\end{tabular} & 16 & 72 Profiles in Bus. \& Mgt. & 2 \\
\hline 73 OneSource & 8 & 73 Promt & 9 \\
\hline 74 PAIS International & 30 & 74 Proquest Direct & 20 \\
\hline \begin{tabular}{l|l}
75 & Political Risk Yearbook \\
\end{tabular} & 8 & \begin{tabular}{|l|l|}
75 & PsycInfo \\
\end{tabular} & 29 \\
\hline 76 Profiles in Bus. \& Mgt. & 2 & 76PWResearcher & 6 \\
\hline 77 Promt (Gale) & 9 & 77 Reference USA & 17 \\
\hline \begin{tabular}{l|l|}
78 & Proquest Direct \\
\end{tabular} & 19 & 78 Research Bank Web & 10 \\
\hline 79 Psyc Articles & 8 & 79 Reuters & 6 \\
\hline 80 Psyc Info & 32 & 80 RIA & 4 \\
\hline 81 Reference USA (Amer. Business Directory) & 15 & \begin{tabular}{l|l|}
81 & S\&P Corporate Text
\end{tabular} & 2 \\
\hline \begin{tabular}{l|l}
82 & Research Bank Web (Investext) \\
\end{tabular} & 17 & \begin{tabular}{l|l|}
82 & S\&P Global Vantage \\
\end{tabular} & 11 \\
\hline 83 Research Insight Global (Global Vantage) & 12 & \begin{tabular}{l|l|}
83 & S\&P NetAdvantage \\
\end{tabular} & 12 \\
\hline 84 Research Insight NA (Compustat PC) & 16 & \begin{tabular}{l|l}
84 & S\&P Research Insight \\
\end{tabular} & 18 \\
\hline 85 Reuters & 11 & 85 SDC & 14 \\
\hline \begin{tabular}{l|l|l}
86 & RIA \\
\end{tabular} & 7 & 86 Social Science Citation Index & 29 \\
\hline 87 S\&P Corporate Text & 1 & 87 Statistical Masterfile & 5 \\
\hline
\end{tabular}




\begin{tabular}{|c|c|c|c|}
\hline $88 \mid$ S\&P NetAdvantage & 17 & 88 Statistical Universe & 25 \\
\hline 89 SafariBooks (Proquest) & 2 & 89 StatUSA & 28 \\
\hline \begin{tabular}{l|l}
90 & SDC (Securities Data Co.) \\
\end{tabular} & 17 & 90 Survey of Buying Power & 2 \\
\hline \begin{tabular}{l|l|}
91 & Source OECD \\
\end{tabular} & 21 & 91 Tablebase & 19 \\
\hline 92 Sports Business Research Network (SBRnet) & 19 & 92 Telerate(Bridge) & \\
\hline 93 STAT USA & 33 & \begin{tabular}{l|l|}
93 & Tradeline \\
\end{tabular} & 2 \\
\hline \begin{tabular}{l|l}
94 & Statistical Masterfile \\
\end{tabular} & 3 & 94 Uncover & 21 \\
\hline \begin{tabular}{l|l}
95 & Statistical Universe \\
\end{tabular} & 33 & \begin{tabular}{l|l}
95 & US Private \\
\end{tabular} & 3 \\
\hline 96 Survey of Buying Power & 2 & 96 US Public & 3 \\
\hline 97 Tablebase (RDS) & 25 & 97 Valueline & 12 \\
\hline \begin{tabular}{l|l}
98 & Tradeline \\
\end{tabular} & 0 & \begin{tabular}{l|l|}
98 & Wilson Bus. Abstracts \\
\end{tabular} & 12 \\
\hline \begin{tabular}{|l|l|}
99 & Uncover \\
\end{tabular} & 11 & \begin{tabular}{l|l}
99 & World Marketing Data \& Statistics \\
\end{tabular} & \\
\hline 100 Value Line & 21 & 100 WorldBank Econ. Indicators & 7 \\
\hline \begin{tabular}{|l|l|}
101 & Web of Science (ISI) \\
\end{tabular} & 31 & \begin{tabular}{|l|l|}
101 & WorldData \\
\end{tabular} & 3 \\
\hline \begin{tabular}{|l|l|}
102 & Wilson Bus. Absts. \\
\end{tabular} & 11 & \begin{tabular}{|l|l|}
102 & Worldscope \\
\end{tabular} & 24 \\
\hline \begin{tabular}{|l|l}
103 & World Marketing Data \& Statistics (Euromonitor) \\
\end{tabular} & 6 & \begin{tabular}{|l|l|}
103 & WSJ \\
\end{tabular} & 21 \\
\hline 104 WorldBank Econ. Indicators & 9 & & \\
\hline \begin{tabular}{|l|l|}
105 & WorldData \\
\end{tabular} & 1 & & \\
\hline \begin{tabular}{|l|l|}
106 & Worldscope (D/Global) \\
\end{tabular} & 19 & & \\
\hline 107 WSJ & 24 & & \\
\hline
\end{tabular}

The survey form also included space for the respondent to add additional databases and aggregations. Thirteen of respondents added eighty unique information resources to the original list. Some of these additions fall outside of the scope of the survey. They include resources from computer science, government, and law. Other additions reflect the unique programs at a particular institution. Many of the "write-in" additions are new products that have been picked up by one of the respondents in the past year. Table 2 is a list of the databases with two or more 'write-in votes.' Business librarians who are unfamiliar with these products may want to investigate them. They may add value to your current offering of databases.

\section{Table 2}

\begin{tabular}{|l|c|}
\hline Additional Databases & $\begin{array}{c}\text { 'Write-in } \\
\text { votes' }\end{array}$ \\
\hline S\&P Execucomp & 5 \\
\hline Faulkner Advisory & 4 \\
\hline Reuters Business Insight & 4 \\
\hline
\end{tabular}




\begin{tabular}{|l|l|} 
TAQ & 4 \\
\hline World Bank Development Indicators & 4 \\
\hline $\begin{array}{l}\text { CCH Internet Research Network (Human } \\
\text { Resources \& labor Law }\end{array}$ & 3 \\
\hline Galante's Capital Private Equity Directory & 3 \\
\hline Global Financial database & 3 \\
\hline MediaMark MRI Reporter & 3 \\
\hline SSRN (Social Science Research Network) & 3 \\
\hline Wall Street Transcript & 3 \\
\hline DRI/ McGraw Hill & 2 \\
\hline EIU Country Commerce & 2 \\
\hline Info Tech Trends & 2 \\
\hline Market Research Monitor & 2 \\
\hline Recap.com & 2 \\
\hline S\&P DRI & 2 \\
\hline
\end{tabular}

\section{The Most Popular Databases and Database Aggregations}

In order to benchmark your libraries electronic business information resources, it is useful to have a ranking of the most widely accessed resources. Table 3 lists all databases and database aggregations listed in the 1999/00 and 2000/01 surveys ranked by the number of libraries providing access. In 2000/01 the responding libraries continued to add database access. The thirty-six libraries in the survey provided access to an average of forty databases in the survey. This is up from an average of thirty-eight databases for thirty libraries in 1999/00.

In 1999/00 top twenty databases were available in twenty-one or more libraries while in 2000/01 the top twenty databases were available in twenty-four or more libraries. To some extent this reflects the increase in the number of respondents from 34 to 36 . If the 80/20 rule, also known as the Pareto Principle, ${ }^{\vee}$ applies to database use, we can hypothesize that these twenty databases account for approximately $80 \%$ of the business database use at these institutions. 
The databases at the bottom of Table 3 are resources that are accessed in only a few libraries. When you have programmatic needs that are not being met by any electronic resources, the bottom of the list is the place to look. One of the responding libraries may have identified a database that will satisfy your unmet need. These selections reflect some unique characteristics of particular library programs. First, some of these libraries are early adapters who have started to access some of the newer products on the market. A few selections from the bottom of this list may move up next year. A second group of the selections from the bottom of the ranked list are databases acquired to meet unique local or programmatic needs. A third category of selections is the expensive databases that are only affordable to select institutions.

Table 2

\section{Academic Business Library Statistics, 2000/2001}

\begin{tabular}{|c|c|c|c|}
\hline Database Ranking, 2000/2001 & $\begin{array}{l}(36 \\
\text { Libraries } \\
\text { reporting) }\end{array}$ & Database Ranking, 1999/2000 & $\begin{array}{l}\text { (34 Libraries } \\
\text { reporting) }\end{array}$ \\
\hline Databases & \begin{tabular}{|l} 
Libraries \\
with \\
Access
\end{tabular} & Databases & $\begin{array}{l}\text { Libraries } \\
\text { with } \\
\text { Access }\end{array}$ \\
\hline 1 EconLit & 34 & $1 \mathrm{ABI} /$ Inform & 33 \\
\hline 2 Lexis-Nexis Academic Universe & 34 & 2 Dow Jones Interactive & 31 \\
\hline 3 JSTOR & 33 & \begin{tabular}{l|l|l|}
3 & JSTOR \\
\end{tabular} & 29 \\
\hline 4 STAT USA & 33 & 4 Lexis-Nexis Academic Universe & 29 \\
\hline 5 Statistical Universe & 33 & 5 PsycInfo & 29 \\
\hline \begin{tabular}{l|l|}
6 Psyc Info \\
\end{tabular} & 32 & 6 Social Science Citation Index & 29 \\
\hline 7 Web of Science (ISI) & 31 & 7 EconLit & 28 \\
\hline 8 Factiva & 30 & 8 StatUSA & 28 \\
\hline 9 PAIS International & 30 & 9 Bloomberg & 27 \\
\hline \begin{tabular}{l|l}
10 & $\mathrm{ABI} /$ Inform Global \\
\end{tabular} & 29 & \begin{tabular}{l|l} 
National Trade Data Bank \\
\end{tabular} & 26 \\
\hline 11 Business \& Industry (RDS) & 28 & 11 Global Access (Primark) & 25 \\
\hline \begin{tabular}{l|l}
12 & NBER Working Papers \\
\end{tabular} & 27 & 12 PAIS International & 25 \\
\hline 13 Bloomberg Financial Markets & 25 & 13 Statistical Universe & 25 \\
\hline 14 Hoovers & 25 & 14CompactDisclosure (Primark) & 24 \\
\hline 15 Investext Plus (Research Bank Web) & 25 & 15 Worldscope & 24 \\
\hline 16 Tablebase (RDS) & 25 & 16 Business \& Industry & 23 \\
\hline \begin{tabular}{l|l|}
17 & Datastream \\
\end{tabular} & 24 & 17| Hoovers & 23 \\
\hline
\end{tabular}




\begin{tabular}{|c|c|c|c|}
\hline 18 Global Access (Primark) & 24 & 18|Datastream & 21 \\
\hline \begin{tabular}{|l|l|}
19 & Lexis-Nexis Classic \\
\end{tabular} & 24 & 19 NBER Working Papers & 21 \\
\hline 20 WSJ & 24 & 20 Uncover & 21 \\
\hline \begin{tabular}{|l|l|}
21 & CRSP \\
\end{tabular} & 23 & \begin{tabular}{l|l|}
21 & WSJ \\
\end{tabular} & 21 \\
\hline \begin{tabular}{|l|l|}
22 & EIU Country reports \\
\end{tabular} & 23 & \begin{tabular}{l|l|}
22 & Investext \\
\end{tabular} & 20 \\
\hline 23 NetLibrary & 23 & \begin{tabular}{l|l|}
23 & Proquest Direct \\
\end{tabular} & 20 \\
\hline 24 FIS Online (Moody's) & 22 & \begin{tabular}{|l|l|l|}
24 & CRSP \\
\end{tabular} & 19 \\
\hline 25 Intl. Financial Statistics & 22 & \begin{tabular}{l|l}
25 & New York Times \\
\end{tabular} & 19 \\
\hline 26 $\mathrm{CCH}$ Internet Tax Service & 21 & \begin{tabular}{l|l|l|}
26 & Tablebase \\
\end{tabular} & 19 \\
\hline 27 Compact Disclosure (Primark) & 21 & \begin{tabular}{l|l|}
27 & Article First \\
\end{tabular} & 18 \\
\hline 28 Source OECD & 21 & \begin{tabular}{l|l}
$28 C H$ & Internet Tax Service
\end{tabular} & 18 \\
\hline 29 Value Line & 21 & \begin{tabular}{l|l|l}
29 & Dialog Web \\
\end{tabular} & 18 \\
\hline 30 ISI Emerging Markets & 20 & \begin{tabular}{l|l|l|}
30 & FIS Online \\
\end{tabular} & 18 \\
\hline 31 MCB-Emerald & 19 & 31 S\&P Research Insight & 18 \\
\hline 32 Proquest Direct & 19 & 32 IFS (International Financial Statistics) & 17 \\
\hline 33 Sports Business Research Network (SBRnet) & 19 & 33 Lexis-Nexis Classic & 17 \\
\hline 34 Worldscope (D/Global) & 19 & 34 Reference USA & 17 \\
\hline 35 Article First & 18 & \begin{tabular}{l|l}
35 & ISI Emerging Markets \\
\end{tabular} & 15 \\
\hline \begin{tabular}{l|l}
36 & Research Bank Web (Investext) \\
\end{tabular} & 17 & 36 Newspaper Abstracts & 15 \\
\hline 37 S\&P NetAdvantage & 17 & 37 SDC & 14 \\
\hline \begin{tabular}{|l|l|}
38 & SDC (Securities Data Co.) \\
\end{tabular} & 17 & \begin{tabular}{l|l|}
38 & Choices II \\
\end{tabular} & 13 \\
\hline 39 Business \& Company Resource Center (Gale) & 16 & 39 Corporate Affiliations Directory & 13 \\
\hline 40 Newspaper Abstracts (Proquest) & 16 & 40 EIU CountryReports & 13 \\
\hline 41 NY Times & 16 & 41 IBES & 13 \\
\hline 42 Research Insight NA (Compustat PC) & 16 & \begin{tabular}{l|l|}
42 & NetLibrary \\
\end{tabular} & 13 \\
\hline 43 Duns MM Directory & 16 & \begin{tabular}{l|l}
43 & Academic Index \\
\end{tabular} & 12 \\
\hline 44 Morningstar & 15 & 44 Bridge & 12 \\
\hline $\begin{array}{ll}45 & \text { Reference USA (Amer. Business Directory) } \\
\end{array}$ & 15 & \begin{tabular}{l|l|}
45 & CorpTech Directory \\
\end{tabular} & 12 \\
\hline 46 Choices II (Simmons) & 14 & 46 Dun's MM Directory & 12 \\
\hline 47 Dialog Web & 13 & 47 Morningstar & 12 \\
\hline 48 EIU Country Data & 13 & 48 S\&P NetAdvantage & 12 \\
\hline 49 FARS & 13 & 49 Valueline & 12 \\
\hline 50 Global Market Information Database (Euromonitor) & 13 & 50 Wilson Bus. Abstracts & 12 \\
\hline 51 IBES & 13 & 51|First Call & 11 \\
\hline \begin{tabular}{l|l|}
52 & Gartner Intraweb \\
\end{tabular} & 12 & \begin{tabular}{l|l|}
52 & S\&P Global Vantage \\
\end{tabular} & 11 \\
\hline 53 Research Insight Global (Global Vantage) & 12 & \begin{tabular}{l|l|}
53 & EBSCO's Business Source \\
\end{tabular} & 10 \\
\hline 54 Corp. Affiliations Directory & 11 & \begin{tabular}{l|l|l}
54 & EIU Views \\
\end{tabular} & 10 \\
\hline 55 Forrester & 11 & \begin{tabular}{l|l}
$55 \mathrm{MCB}$ (Emerald) \\
\end{tabular} & 10 \\
\hline 56 General Business File & 11 & \begin{tabular}{|l|l|}
56 & Political Risk \\
\end{tabular} & 10 \\
\hline \begin{tabular}{l|l|l}
57 & Reuters \\
\end{tabular} & 11 & \begin{tabular}{l|l}
57 & Research Bank Web \\
\end{tabular} & 10 \\
\hline 58 Uncover & 11 & 58 EIU Country Data & 9 \\
\hline 59 Wilson Bus. Absts. & 11 & \begin{tabular}{|l|l|}
59 & GAPRO (Primark) \\
\end{tabular} & 9 \\
\hline \begin{tabular}{l|l}
60 & Academic Index \\
\end{tabular} & 10 & \begin{tabular}{l|l}
60 & General Business file \\
\end{tabular} & 9 \\
\hline \begin{tabular}{l|l|}
61 & CareerSearch \\
\end{tabular} & 10 & 61 Infotrac Online & 9 \\
\hline 62 EBSCO Business Source Premier & 10 & 62 Promt & 9 \\
\hline 63 Infotrac (IAC) & 10 & 63 Business \& Management Practices & 8 \\
\hline \begin{tabular}{l|l|l|l|l}
64 & First Call \\
\end{tabular} & 9 & $64 \mid$ EIU News & 8 \\
\hline
\end{tabular}




\begin{tabular}{|c|c|c|c|}
\hline 65 GA Pro (LaserD, Primark) & 9 & 65 Kalorama (MarketResearch.com) & 8 \\
\hline 66 Jupiter & 9 & 66 Business Periodicals Index & 7 \\
\hline 67 Promt (Gale) & 9 & 67 Career Search & 7 \\
\hline 68 WorldBank Econ. Indicators & 9 & 68 Economic Literature DB & 7 \\
\hline \begin{tabular}{l|l}
69 & Bridge/Telerate \\
\end{tabular} & 8 & 69 FARS & 7 \\
\hline 70 Business \& Mgt. Practices & 8 & 70 Gale & 7 \\
\hline 71 EBSCO Business Source Elite & 8 & 71 OneSource & 7 \\
\hline 72 EIU Business Intl newsletters & 8 & 72 WorldBank Econ. Indicators & 7 \\
\hline 73 OneSource & 8 & 73 Accounting \& Tax Index & 6 \\
\hline 74 Political Risk Yearbook & 8 & 74 Business Periodicals Ondisc & 6 \\
\hline 75 Psyc Articles & 8 & 75 Gartner & 6 \\
\hline \begin{tabular}{l|l}
76 & $\mathrm{ABI} /$ Inform Research \\
\end{tabular} & 7 & 76 National Newspaper Index & 6 \\
\hline 77 Business Periodicals Index & 7 & 77 PWResearcher & 6 \\
\hline \begin{tabular}{l|l|}
78 & EIU Views Wire \\
\end{tabular} & 7 & 78 Reuters & 6 \\
\hline 79 Market Insight (S\&P) & 7 & \begin{tabular}{l|l|}
79 & Citibase \\
\end{tabular} & 5 \\
\hline \begin{tabular}{l|l|}
80 & Multex \\
\end{tabular} & 7 & \begin{tabular}{l|l|l|}
$80 x t e l$ \\
\end{tabular} & 5 \\
\hline \begin{tabular}{l|l}
81 & RIA \\
\end{tabular} & 7 & 81 Global Market Information & 5 \\
\hline \begin{tabular}{l|l}
82 & Accounting \& Tax Index \\
\end{tabular} & 6 & \begin{tabular}{l|l|l|}
82 & OECD Index \\
\end{tabular} & 5 \\
\hline \begin{tabular}{l|l|}
83 & Comperio \\
\end{tabular} & 6 & \begin{tabular}{l|l|}
83 & Statistical Masterfile \\
\end{tabular} & 5 \\
\hline \begin{tabular}{l|l|}
84 & Economic Literature DataBase \\
\end{tabular} & 6 & \begin{tabular}{l|l}
84 & Telerate(Bridge) \\
\end{tabular} & 5 \\
\hline \begin{tabular}{l|l|}
85 & Gale Business Resources \\
\end{tabular} & 6 & \begin{tabular}{l|l|l|}
$85 \& S$ & Index \\
\end{tabular} & 4 \\
\hline 86 MarketResearch.com & 6 & 86 Nikkei & 4 \\
\hline 87 World Marketing Data \& Statistics (Euromonitor) & 6 & 87 RIA & 4 \\
\hline 88 Books $24 \times 7$ & 5 & 88 World Marketing Data \& Statistics & 4 \\
\hline 89 Business Index (Gale) & 5 & 89 Corporate Profiles & 3 \\
\hline 90 Business Periodicals Ondisc & 4 & 90 DataStar & 3 \\
\hline \begin{tabular}{l|l|}
91 & Citibase \\
\end{tabular} & 4 & \begin{tabular}{l|l|}
91 & DataTimes \\
\end{tabular} & 3 \\
\hline \begin{tabular}{l|l|}
92 & Duns Business Locator \\
\end{tabular} & 3 & 92 US Private & 3 \\
\hline 93 National Newspaper Index (IAC) & 3 & 93 US Public & 3 \\
\hline 94 Statistical Masterfile & 3 & \begin{tabular}{l|l|}
94 & WorldData \\
\end{tabular} & 3 \\
\hline \begin{tabular}{l|l}
95 & Data Star \\
\end{tabular} & 2 & \begin{tabular}{l|l|}
95 & Amer. Manufacturers Directory \\
\end{tabular} & 2 \\
\hline \begin{tabular}{l|l|}
96 & Data Times \\
\end{tabular} & 2 & 96 Business Index & 2 \\
\hline 97 F\&S Index (Gale) & 2 & 97 Business InfoSuite (Gale) & 2 \\
\hline 98 Nikkei & 2 & 98 Profiles in Bus. \& Mgt. & 2 \\
\hline 99 Profiles in Bus. \& Mgt. & 2 & \begin{tabular}{|l|l}
99 & S\&P Corporate Text \\
\end{tabular} & 2 \\
\hline 100 SafariBooks (Proquest) & 2 & \begin{tabular}{|l|l|}
100 & Survey of Buying Power \\
\end{tabular} & 2 \\
\hline 101 Survey of Buying Power & 2 & 101 Tradeline & 2 \\
\hline 102 Amer. Mfgrs. Directory & 1 & 102 Dun's Business Locator & 1 \\
\hline \begin{tabular}{|l|l|}
103 & Business Infosuite (Gale) \\
\end{tabular} & 1 & 103 Phonedisc & 1 \\
\hline \begin{tabular}{l|l|}
104 & Extel \\
\end{tabular} & 1 & & \\
\hline \begin{tabular}{|l|l|}
105 & S\&P Corporate Text \\
\end{tabular} & 1 & & \\
\hline 106 WorldData & 1 & & \\
\hline \begin{tabular}{|l|l|}
107 & Tradeline \\
\end{tabular} & 0 & & \\
\hline
\end{tabular}

\section{Summary}


This report presents survey data on access to databases and database aggregations. The value of the information presented in this article lies in the comparison of the ranked lists presented above with the reader's institutional access. A review of the databases at the bottom of the ranked list may turn up a potential candidate to meet unique library needs. With the development of the Internet, the development of electronic information and the distribution and access to this information is changing rapidly. This survey points out that academic business libraries are moving to take advantage of the new content and new functionality that is being developed by information providers.

\section{NOTES}

${ }^{\mathrm{i}}$ Aggregations are compilations of electronic resources, data files, reports, journals and other electronic information. Aggregations meet two criteria. First, they are distributed by a single agency or group (an aggregator) with responsibility for maintaining the collection and selecting resources to be included in the aggregation. Second, they are prepared and distributed as a collection. See, William H. Walters, Samuel G. Demas, Linda Stewart and Jennifer Weintraub. Guidelines for Collecting Aggregations of Web Resources. (Working Paper) Albert R. Mann Library, Cornell University, Ithaca, N.Y. 14853, [1998].

ii Davis, Trisha L., "The evolution of selection activities for electronic resources." Library Trends 45(3) Winter 1997:391-403.

iii Holleman, Curt, "Electronic resources: are basic criteria for the selection of materials changing?" Library Trends 48(4) Spring 2000:694-710.

iv Thornton, Glenda A., "Impact of electronic resources on collection development, the roles of librarians and library consortia." Library Trends 48(4) Spring 2000:842-856.

v The Pareto Principle was popularized by Joseph Juran. See, Zairi, Mohamed, "Juran, Jospeh M." in International Encyclopedia of Business \& Management 3:2246-2247, edited by Malcolm Warner. New York: Routledge, 1996. 